\title{
Polarization and Depression-Angle Dependence of Radar Terrain Return
}

\author{
I. Katz and L. M. Spetner
}

(March 25, 1960; revised May 2, 1960)

\begin{abstract}
A study of recent experimental results on radar back scattering from land and sea surfaces indicate: (a) The polarization dependence of the normalized radar cross section, $\sigma_{0}$, of ocean surfaces cannot be explained by the usual "interference phenomenon," and (b) there is a distinct difference in the form of the depression-angle dependence in that $\sigma_{0}$ for "smooth" surfaces follows a negative exponential whereas $\sigma_{0}$ for "rough" surfaces drops off as the sine of the depression angle.
\end{abstract}

\section{Introduction}

In order to progress toward a better understanding of back-scattering from natural surfaces a large amount of radar-return data from various sources has been studied.

For the first time in the history of the radar terrainreturn problem, large volumes of data have become available. The data which have been studied include experimental results from the Naval Research Laboratory and Goodyear Aircraft Corp. under sponsorship of the Applied Physies Laboratory, Ohio State University, and the Admiralty Signal and Radar Establishment and the Royal Radar Establishment in Great Britain. As a whole, these data include radar return from various kinds of terrain as well as from the ocean in various sea states, several wave lengths, and in general both polarizations. In most of these cases, a wide range of depression angles has been covered. As a result of these studies certain features of radar terrain back-scattering are becoming clear. In this paper the polarization and depression-angle dependence of radar return are discussed.

\section{Polarization Dependence}

The polarization dependence of sea return is more striking than that of land return and the present discussion of polarization dependence will be confined to sea return. Herbert Goldstein [1] ${ }^{2}$ has postulated a droplet theory to explain the fact that the radar return for horizontal polarization is less than that for vertical polarization. In his theory, it is the droplets or spray particles that are cast up by the water which do most of the reflecting or backscattering of the radar energy. Since these droplets are illuminated within the interference pattern

1 Contribution from Applied Physics Laboratory, The Johns Hopkins Uni. versity, Silver Spring, Md. This work was supported by the Bureau of Naval versity, Silver Spring, Md. This work was supported by the B
Weapons, Department of the Navy under Contract NOrd-7386.

2 Figures in brackets indicate the literature references at the end of this paper. formed by the direct ray from the radar and the reflection from the ocean surface, horizontally polarized radiation which has a higher reflection coefficient for forward scattering has a deeper first null and hence, gives weaker illumination on the scattering droplets. This leads to a smaller radar return. Katzin [2] has postulated surface scatterers in the place of Goldstein's droplets, which are also illuminated within the interference pattern of the radar.

There is now evidence that the polarization dependence of sea return cannot be explained by an interference pattern in the illumination. Results on forward scattering of energy by the ocean surface [3] indicate that for microwave frequencies and for depression angles above a few degrees in most sea conditions the interference pattern may be negligible even for horizontal polarization. In figure 1 are shown experimental values of the normalized radar cross section, $\sigma_{0}$, plotted against depression angle using an L-band radar. Although the sea state in this experiment is unknown, the water surface was subjected to a $30-k n o t$ wind which would normally result in $8 \mathrm{ft}$ rms wave heights in a fully developed sea. Interference effects, at depression angles above $10 \mathrm{deg}$, can account for less than $3 \mathrm{db}$ difference bet,ween the reflected power for horizontal and vertical polarization even if one assumes only a root mean square wave height of $1 \mathrm{ft}$. Yet the difference between the powers returned on the two polarizations is greater than $6 \mathrm{db}$ between 10 and $25 \mathrm{deg}$.

It is only at the very low angles that significant interference effects can arise, usually at angles substantially smaller than $1 \mathrm{deg}$, depending, of course, upon the sea state and the wave length. Note, again in figure 1, that at the lower depression angles it appears that the curve for vertical polarization is tending to cross the one for horizontal polarization. Figure 2 [4] shows that this trend continues and crossover does take place, ${ }^{3}$ i.e., for depression angles

${ }^{3}$ Although figures 1 and 2 refer to different sea states and different radar frequencies they do tend to establish the existence of crossover at least for some
frequencies and for some sea states. 


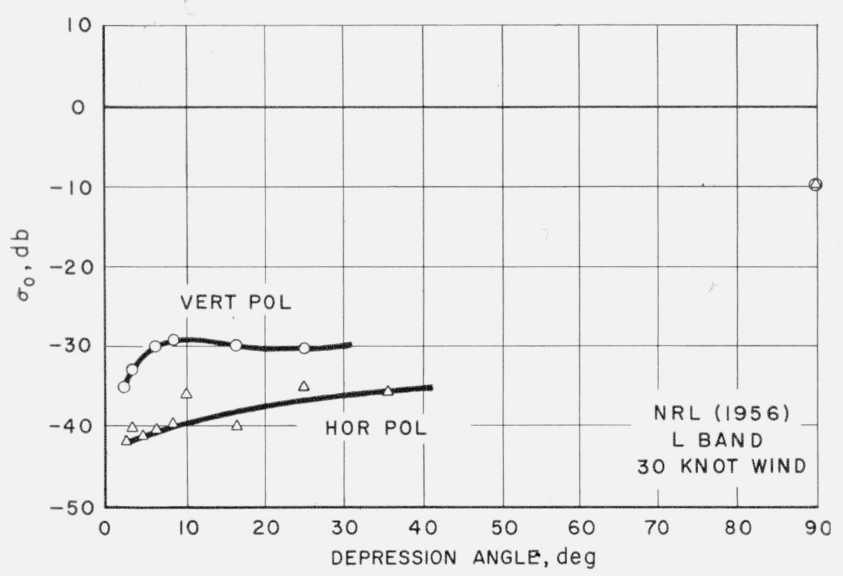

Figure 1. Normalized radar cross section of sea surface for vertical and horizontal polarizations as a function of depression angle.

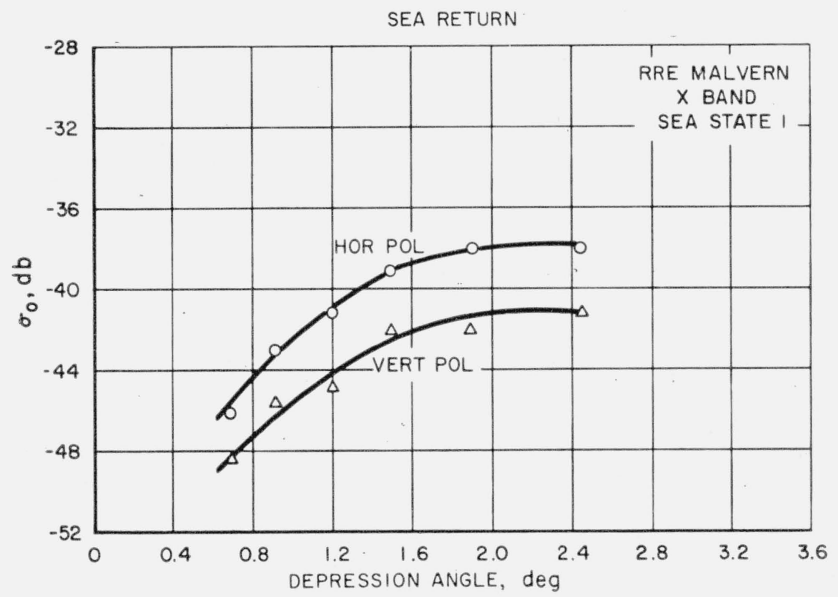

Figure 2. Normalized radar cross section of sea surface for vertical and horizontal polarizations as a function of depression angle for very small depression angles.

less than $2.5 \mathrm{deg}$ the normalized radar cross section for vertical polarization is smaller than that for horizontal polarization.

Thus the interference pattern explanation of the polarization dependence of sea return is faced with the following dilemma. It serves as an explanation of the fact that $\sigma_{0}$ for vertical polarization is greater than that for horizontal polarization but a nonnegligible interference pattern obtains only at extremely low depression angles. At these low angles, however, experimental evidence is quite to the contrary in that $\sigma_{0}$ for vertical polarization is smaller than that for horizontal polarization. Where the experimental data show $\sigma_{0}$ for vertical polarization greater than that for horizontal polarization, interference theory is at a loss for an explanation.

The interference theory has a further unsatisfactory feature. It is not clear how a scatterer on the surface can be illuminated in an interference pattern which includes a ray reflected from the same surface. If the scatterers on the surface of a homogeneous sea are sufficiently illuminated to enable them to scatter in the forward direction a ray which is strong enough to produce a deep null in the interference pattern, then the scatterers should be illuminated strongly enough to reflect back to the radar.

Since the interference phenomenon cannot reasonably explain the polarization dependence of sea return one must look elsewhere; it is possible that the correct explanation will come by examining the fundamental properties of electromagnetic scattering from an ocean-type surface. Katzin suggested in his paper an array of circular disks as a model for the ocean surface. The exact theory of electromagentic scattering off a circular disk has recently been worked out by Flammer [5] and by Meixner and Andrejewski [6]. The results of these calculations are difficult to evaluate numerically. Calculations have been made, however, from Flammer's results for a disk of diameter of the order of a wavelength. These results show that a disk has a larger backscattering cross section for horizontal than for vertical polarization. This agrees with the results of Copson [7] for scattering from an extremely small disk. In applying these results to interpretation of the data it is tacitly assumed that effects of multiple scattering are negligible. It is interesting to note that single scattering off small circular disks is consistent with the experimental results available at the extremely low angles, because at the very low angles one expects only the small and rather isotropic scatterers to be effective. At the higher angles, one can expect larger, more directive, scatterers to contribute the bulk of the radar return; an examination of the back-scattering from a large circular disk may suggest an appropriate explanation for the experimental results.

\section{Depression-Angle Dependence}

Data from Ohio State University [8] on radar return from various kinds of land surfaces show that for the case of rough surfaces with only a few exceptions, there seems to be no significant difference between horizontal and vertical polarization, but for smooth surfaces and for angles larger than $10 \mathrm{deg}$, $\sigma_{0}$ for vertical polarization is larger than that for horizontal polarization just as in the case of the ocean.

This leads to a plausible explanation for the depression angle dependence of sea return. At extremely large depression angles, near $90 \mathrm{deg}$, the radar return arises largely from specular reflections off the very large and almost horizontal facets of the ocean surface. At the very small depression angles, radar return is in large part caused by the isotropic scattering off the extremely small scatterers. In the intermediate range of depression angles from about 20 to $70 \mathrm{deg}$, lies an interesting region which may also hold the key to the polarization dependence.

$\dot{A}$ study of the curves of normalized radar cross section versus depression angle, $\theta$, brings out clearly the difference in form between "rough" and "smooth" surfaces. For the most part the curves taken over rough surfaces show that $\sigma_{0}$ varies as $\theta$ or $\sin \theta$ between 10 and 80 deg. Figure 3 is an example of 


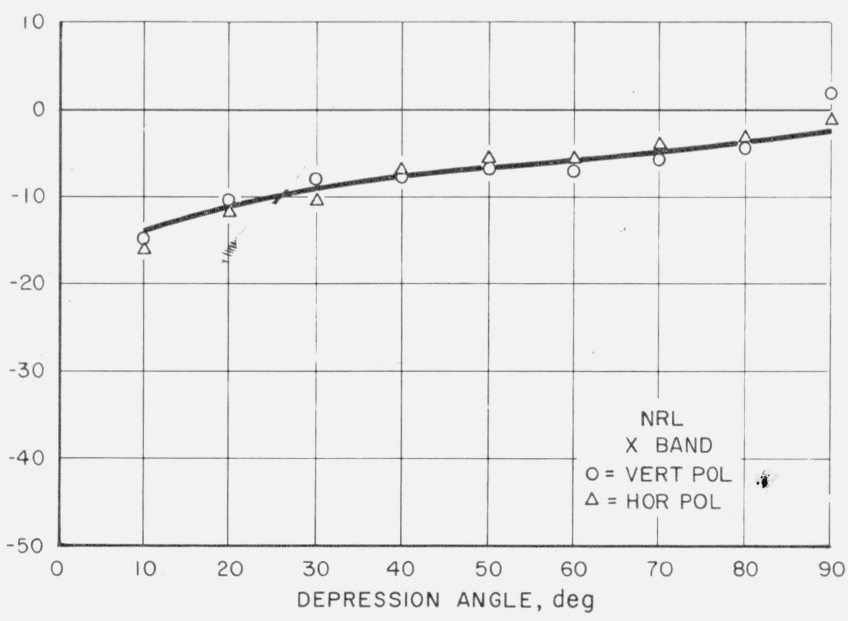

Figure 3. Normalized radar cross section of forest.

The solid line represents a linear variation of $\sigma_{0}$ with $\theta$.

radar return from a forested area using an X-band radar. These measurements were made over New Jersey woods which consisted of trees about $50 \mathrm{ft}$ high. The important feature of the radar return from rough terrain appears to be the flatness of the $\sigma_{0}$ versus $\theta$ curves.

For smoother surfaces the cross section curves increase more sharply with increasing depression angle as illustrated in figure 4 . In this figure are presented radar-return data from three surfaces with different degrees of smoothness characterized as: (1) concrete road, (2) concrete road with 2 in. of smooth snow, and (3) concrete road with 2 in. of rough snow; clearly, the smoother the surface the steeper is the $\sigma_{0}$ curve.

In a previous paper [9] theoretical results were reported based on a facet model with specular reflection to explain radar return from smooth-type surfaces. There it was suggested that if the probability distribution of the slopes were Gaussian one might expect that the radar cross section would be proportional to $\exp \left(-k \cot ^{2} \theta\right)$. An examination of the experimental results that have since become

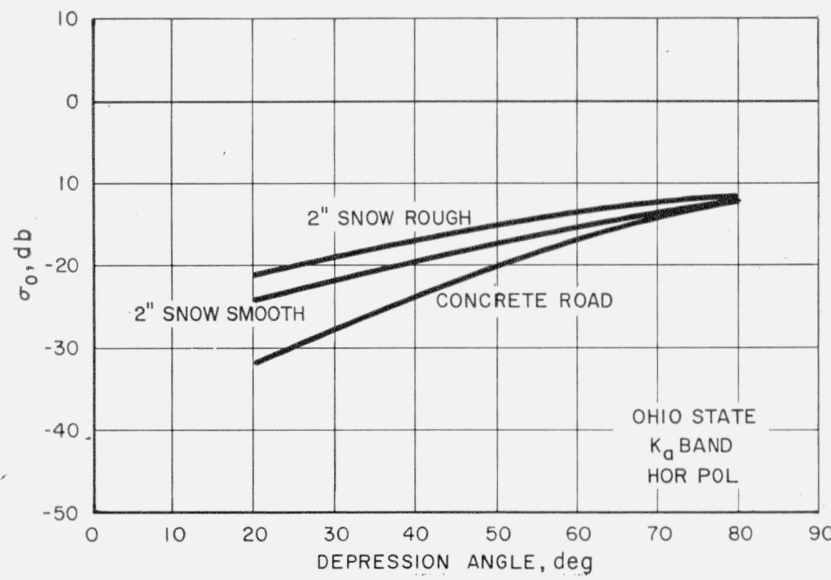

Figure 4. Normalized radar cross section showing the transition from rough to smooth suifaces. available indicates that a better description of the radar cross section would be given by $\exp (-k \cot \theta)$ instead. This suggests that if the model of a random distribution of facet slopes is to be preserved then the probability distribution of the slope, $x$, is of the form $\exp (-k x)$ rather than a Gaussian. Figure 5 is an example of data taken over Lake Michigan using both polarizations which show the depression angle regions where the form $\exp (-k \cot \theta)$ for $\sigma_{0}$ holds.

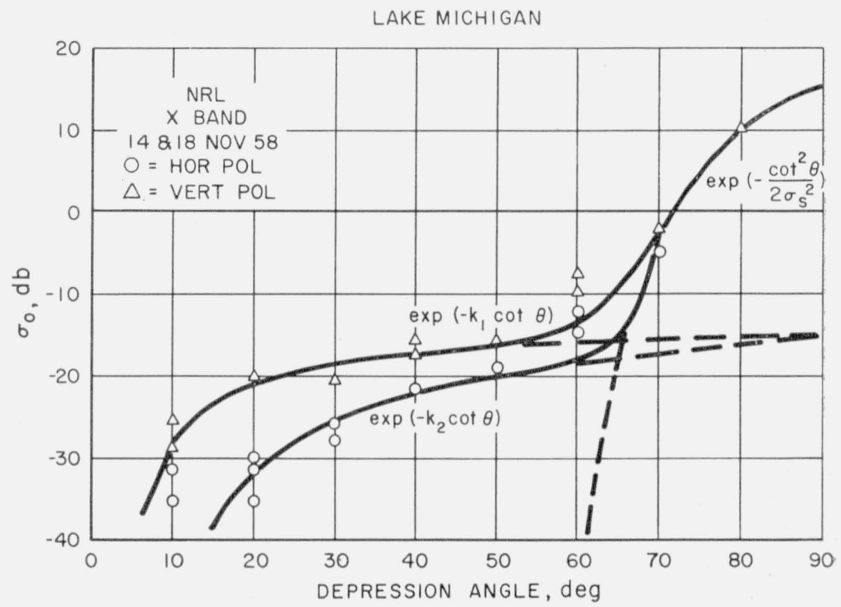

Figure 5. Normalized radar cross section of the surface of Lake Michigan for horizontal and vertical polarizations.

In the case of extremely low angles over land a mechanism was previously suggested [10] that would lead to an increase in $\sigma_{0}$ as $\theta$ approaches very close to zero. This mechanism was based on more efficient scattering from vertical structures as the depression angle approaches zero. It is noteworthy that data for extremely small angles between 1 and $4 \mathrm{deg}$ show there is an upturn in the curves with decreasing $\theta$. An example of this is shown in figure 6 .

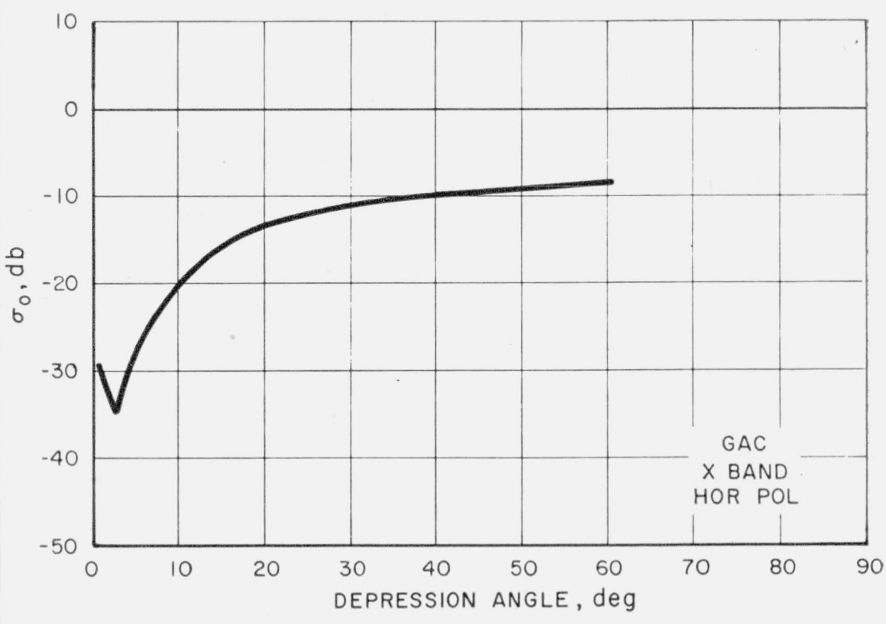

FIGURE 6. Normalized radar cross section of cultivated terrain illustrating the increase of $\sigma_{0}$ with decreasing $\theta$ for very small values of depression angle. 


\section{Conclusion}

Another mechanism must be found to explain the polarization dependence of sea return. It would be instructive to calculate the back scattering from disks having diameters of a wavelength and greater. The non-Gaussian nature of the reflecting facets in the ocean surface is somewhat surprising. This fact may point to a clearer understanding of radar reflectivity, if pursued.

\section{References}

[1] D. E. Kerr, Propagation of Short Radio Waves, pp 481527 (McGraw-Hill Book Co., Inc., New York, N.Y., 1951)

[2] M. Katzin, On the mechanisms of radar sea clutter, Proc. IRE 45, 44 (1957).

[3] C. I. Beard, I. Katz, L. M. Spetner, Phenomenological vector model of microwave reflection from the ocean, IRE Trans. AP-4, 162 (1956).
[4] Private communication from M. H. Oliver, (Oct. 6, 1959).

[5] C. Flammer, The vector wave function solution of the diffraction of electromagnetic waves by circular disks and apertures, J. Appl. Phys. 24, 1218 (1953).

[6] J. Meixner and W. Andrejewski, Exact theory of the diffraction of plane electromagnetic waves from a perfectly conducting circular disk and from a circular aperture in a perfectly conducting plane screen (in German), Ann. Phys. \%, 157 (1950).

[7] E. T. Copson, An integral-equation method of solving diffraction problems, Proc. Roy. Soc. (London) $[\mathbf{A}]$ 186, 100 (1946)

[8] R. C. Taylor, Terrain return measurements at $\mathrm{X}$-, $\mathrm{K}_{u^{-}}, \mathrm{K}_{a}$-band, IRE Conv. Rec. I (1959).

[9] L. M. Spetner and I. Katz, Two statistical models for radar terrain return, IRE Trans. AP-8, (May 1960).

10] I. Katz and L. M. Spetner, A program to investigate * radar terrain return, The Johns Hopkins Univ. Appl. Phys. Lab. Rept. CF-2700 (1958)

(Paper 64D5-83) 